Instituto Internacional de Investigación y Desarrollo Tecnológico Educativo INDTEC, C.A.

DOI: https://doi.org/10.29394/Scientific.issn.2542-2987.2020.5.18.8.157-172

OAI-PMH: http://www.indteca.com/ojs/index.php/Revista Scientific/oai

Artículo Original / Original Article

\title{
La vinculación social y la seguridad ciudadana: Un transcategorial emergente de la Seguridad
}

\author{
Autor: Luis Enrique Rodríguez Velazco \\ Universidad Nacional Experimental de la Seguridad, UNES \\ abg.luisrodriguez@gmail.com \\ Maracay, Venezuela \\ https://orcid.org/0000-0002-7680-6348
}

Resumen

La experiencia profesional y laboral en la Universidad Nacional Experimental de la Seguridad (UNES), en materia social y en especial en el área de vinculación social permitieron ver, sentir la necesidad de construir una nueva conceptualización de vinculación social en optimizar la seguridad ciudadana. La presente investigación es de enfoque cuali-cuantitativo, apoyado en el Multimétodo: investigación acción (IA) y Hermenéutico Critico. El objetivo es construir un conocimiento transcomplejo sobre la vinculación social para la optimización de la seguridad ciudadana. La metodología se centró en la recopilación de información mediante la observación participante, entrevista con informantes claves y la participación directa del investigador con el objeto de estudio, en relación a la entrevista con informantes claves se realizaron a tres (3) grupos de investigadores: Un (1) Investigador en materia de Seguridad Ciudadana, un (1) investigador en transcomplejidad y un (1) investigador en Vinculación Social. Se tiene como propósito la construcción del conocimiento transcomplejo sobre la vinculación social en la optimización de la seguridad ciudadana, crear nuevas epistemes, además de responder los planteamientos que permitan irrumpir la visión Transcompleja sobre la vinculación social, se orientan los referentes teóricos para resaltar la validez, su aplicabilidad en la metodología, sus aportes deben incorporar la transcomplejidad como constructo de conocimientos sobre la vinculación social.

\section{ciudadana.}

Palabras clave: transcomplejidad; vinculación social; seguridad

Cómo citar este artículo:

Rodríguez, L. (2020). La vinculación social y la seguridad ciudadana: Un transcategorial emergente de la Seguridad. Revista Scientific, 5(18), 157-172, e-ISSN: 2542-2987. Recuperado de: https://doi.org/10.29394/Scientific.issn.2542-2987.2020.5.18.8.157-172

Fecha de Recepción: 23-05-2020
Fecha de Aceptación: 28-09-2020
Fecha de Publicación: 05-11-2020 
OAI-PMH: http://www.indteca.com/ojs/index.php/Revista_Scientific/oai

Artículo Original / Original Article

\title{
Social bonding and citizen security: An emerging transcategorial of Security
}

\begin{abstract}
The professional and work experience at the National Experimental University of Security (UNES), in social matters and especially in the area of social bonding, allowed us to see and feel the need to build a new conceptualization of social bonding in optimizing citizen security. The present investigation is of a quali-quantitative approach, supported by the Multimethod: action investigation (IA) and Critical Hermeneutics. The objective is to build a transcomplex knowledge about social bonding for the optimization of citizen security. The methodology focused on the collection of information through participant observation, interview with key informants and the direct participation of the researcher with the object of study, in relation to the interview with key informants, three (3) groups of researchers were carried out: A (1) Researcher on Citizen Security, one (1) researcher on transcomplexity and one (1) researcher on Social Bonding. The purpose is to build transcomplex knowledge about social bonding in the optimization of citizen security, create new epistemes, in addition to responding to the approaches that allow the transcomplex vision of social bonding to break out, the theoretical referents are oriented to highlight the validity, its applicability in the methodology, its contributions must incorporate transcomplexity as a construct of knowledge about social bonding.
\end{abstract}

Keywords: transcomplexity; social bonding; citizen security.

\footnotetext{
How to cite this article:

Rodríguez, L. (2020). Social bonding and citizen security: An emerging transcategorial of Security. Revista Scientific, 5(18), 157-172, e-ISSN: 2542-2987. Recovered from: https://doi.org/10.29394/Scientific.issn.2542-2987.2020.5.18.8.157-172

Date Received:

Date Acceptance:

Date Publication:
} 23-05-2020
28-09-2020
05-11-2020 


\section{Introducción}

La Universidad Nacional Experimental de la Seguridad (UNES) desde su creación está ligada a los principios de formación enmarcados en el Documento Fundacional de la (UNES, 2010a): plantea que la Transdisciplinariedad donde hace referencial conceptual: el estudio de un fenómeno desde varias disciplinas a través de ellas, pongamos un ejemplo claro para adentrarnos en la investigación: Problemas en materia de seguridad ciudadana, estos deben ser enfocados desde la sociología, pedagogía, criminología, así mismo construir nuevos conocimientos que originados con el apoyo de la transdisciplinariedad y la complementariedad. De allí emerge la transcomplejidad como primera categoría de la investigación, este nuevo conocimiento en materia de seguridad es novedoso, intelectuales han plantado en sus tesis la transcomplejidad como método de producción de conocimiento por la amplitud de comprender y reflexionar antes el objeto de estudio a desarrollarse.

Es menester desarrollar este método por su producción epistémica nos abrirá un abanico de orientaciones en la vinculación social siendo otra categoría reveladora y articuladora en la comunidad y factor determinante para realizar abordajes comunitarios, esta vinculación social permite conocer la realidad social de manera directa y personal, sin que este fenómeno sea cambiado desde su propia subjetividad, la seguridad ciudadana debe arropar la realidad social con su manto epistémico a través de la transcomplejidad como un universo de conocimientos y la vinculación social por su cálido contacto permanente en la sociedad.

Comprendiendo esta disciplina y anexando las categorías, podemos aportar valiosas soluciones a tan compleja situación nacional e internacional como es el flagelo de la inseguridad, que tanto daño hace a la sociedad. Esta complejidad que por ser tan dispersas hay que verla con la óptica de la transcomplejidad para desenlazar las causas y consecuencias para lograr una 
optimización de la seguridad ciudadana. A hora bien nos podemos preguntar: ¿Cuál es la caracterización actual de la vinculación social en la UNES?; ¿La UNES estará abierta a nuevos aportes transcategorial?; ¿Qué se necesita para incorporar el modelo transcomplejo en las nuevas investigaciones?. Las Interrogantes expuestas, se presentan en la investigación para lograr la comprensión de la realidad social e incorporación transcategorial en la optimización de la seguridad ciudadana.

\subsection{Abordaje Teórico}

En Venezuela, como en muchos países poseen una modelo legal, con características similares a temas de seguridad ciudadana, social y político entre otros. En el país, nuestro marco legal y máxima jerarquía suprema se llama Constitución de la República Bolivariana de Venezuela (1999): en la cual se establece que: "toda persona tiene derecho a la protección por parte del Estado a través de los órganos de seguridad ciudadana regulados por ley, frente a situaciones que constituyan amenaza, vulnerabilidad o riesgo para la integridad física de las personas [...]" (art. 55); a hora bien, frente a posibles situaciones que constituyan amenazas, es necesario decir que los órganos de seguridad ciudadana de Venezuela, como Cuerpos de Policías, Cuerpo de Investigaciones Científicas y Criminalísticas (CICPC), Bomberos, Protección Civil y Guardas Parques, entre otros órganos para la protección de la sociedad y combatir estas amenazas, las podemos considerar como parte de una inseguridad y a la vez se puede ver como un nido que empieza a crecer y que formará una delincuencia.

Este flagelo perturbador en nuestra sociedad moderna tan compleja y complicada que abordaremos en tres categorías emergentes, de las cuales la primera de ellas es: La Transcomplejidad que aborda Villegas y Schavino (2012), citados por Alfonzo, et al. (2018): "[...] constituye un concepto categorial emergente, propio de la epocalidad, amparada en la 
posmodernidad, en la cual confluyen de manera sinérgica las categorías epistémicas de complejidad y la transdisciplinariedad [...]” (pág. 78).

Indiscutiblemente el ser humano mientras se desarrolla es cada día más complejo, y esta complejidad se mantiene en orden cuando somos ciudadanos ejemplares para el bien de la sociedad, entonces al contrario sería el desorden el ciudadano perturbador de la sociedad. La transcomplejidad, pensamiento que actualmente es utilizado en distintas Universidades en el ámbito de post grados me permitió orientar esta investigación transcategorial emergente para construir un pensamiento a través la transdisciplinariedad.

Es decir, una articulación de varias disciplinas para el estudio de una realidad. Una de ellas en este artículo es la sociología, ciencia que estudia las relaciones sociales. Allí emerge la vinculación social sustentada en nuestro Documento Fundacional de la Universidad Nacional Experimental de la Seguridad (UNES, 2010b): "[...] es entendida como el proceso de relacionamiento entre sujetos colectivizados (universidad-comunidad) que desde su ser, su hacer y su saber, se integran de manera proactiva para convivir [...]" (pág. 37). Esa relación compleja entre una comunidad y la universidad para abordar la realidad social es ontología existente que diariamente se compleja y abrumadora sino se acompaña con la óptica de la transdisciplinariedad como principio de formación.

Estudiándola desde lo epistemológico, sociológico, lo ontológico, esta sociedad y UNES, construirán nuevos pensamientos de relaciones para satisfacer necesidades. Estas carencias en la actualidad la definimos motivación de seguridad, Venegas (2019): esboza "la motivación no se caracteriza como un rasgo personal, sino por la interacción de las personas frente situación que se desea alcanzar, por ejemplo el hábito lector" (pág. 16). Observando que cubierta la primera necesidad que es las fisiológicas el individuo, pasa a un segundo eslabón de necesidad que es la de seguridad: esta principalmente de salud, vivienda, protección. 
Esta que debe garantizar el estado establecido en el artículo constitucional antes prenombrado. Sin embargo, abordando el termino de seguridad ciudadana y experiencia en materia de vinculación social no solamente debe ser responsable el estado debemos mirar esa responsabilidad en conjunto por la complejidad de la misma, debemos observarla desde la complejidad como principio y enfocarlas desde distintas disciplinas. Como respuesta a las incertidumbres hechas al principio encontramos certidumbres a través de los Principios de Formación UNES, como la dialogicidad y transdisciplinariedad.

Entre otros importantes principios que nos conduce el primero a prácticas reales al compartir ese discernimiento diario en la sociedad, y el otro la aplicabilidad compleja de las disciplinas en su estrecha relación es decir ver la seguridad ciudadana a través de la vinculación social para construir un pensamiento transcomplejo.

También están presentes los Principios hacedores de nuestra investigación científica que permite estudiar la seguridad ciudadana desde lo ontológico, lo axiológico lo epistemológico, que se constituye como una realidad social con sus niveles de necesidades, dicho así, entre la comunidad y los niveles de necesidades, al respecto hay que tomar en consideración a Simón Bolívar (1783-1830), como precursor de la educación crítica y la educación emancipadora como lo menciona Rumazo (2006): "los pocos que llegan a sobresalir intelectualmente, o han sido enviados a educarse en Europa -como se hará con Simón Bolívar-, o han profundizado estudios por propia iniciativa, superando toda suerte de dificultades" (pág. 12). Hablar de vinculación social, en un estrecho horizonte complejo de investigaciones populares.

Ahora retomando a las respuestas, claro está que la Universidad Nacional Experimental de la Seguridad, en su caracterización actual de la vinculación social, está impregnada en nuestros pensadores latinoamericanos, 
en su accionar social, Es apropiado disipar que en esta sociedad tan compleja en materia de seguridad ciudadana, debemos identificar los fundamentos sociológico y axiológico de una sociedad con interrelación a la UNES, estos fundamentos por ende serán complejizados en el abordaje metodológico, para comprender y recomprender la sociedad y su vínculo social. Está abierta a nuevos cambios transcategorial y desde el inicio la universidad se considera bajo el Documento Fundacional de la Universidad Nacional Experimental de la Seguridad (UNES, 2010c): en el que se rigen por los principios de transdisciplinariedad y dialogo.

Estos Principios fundamentales que muy bien nos brindan horizontes universales, favoreciendo la intercolaboración y retroalimentación entre la comunidad UNESISTA y la sociedad, es un nacimiento de un conocimiento nuevo con potencialidad de aprender, reaprender, construir, deconstruir, visibilizar el orden y enfocar el desorden, estos últimos forman parte compleja del dialogo. ¿Qué se necesita para incorporar el método transcomplejo a las nuevas investigaciones?, más allá de investigadores metacomplejos necesariamente con voluntades transcategoriales, bajo los principios de la complejidad y la transdisciplinariedad. En este orden de ideas, es necesario exponer los fundamentos ontológicos, axiológicos, epistemológicos y metodológicos de esos aportes teóricos.

\subsection{Fundamento ontológico}

Lo ontológico se refiere a la naturaleza de los fenómenos sociales, mirar la seguridad ciudadana a través de la transcomplejidad entonces nos revelara la naturaleza de la realidad, esta realidad de la seguridad objetiva que siente la comunidad o subjetiva que observa en las necesidades de una comunidad, de allí la importancia de la fundamentación ontológica. 


\subsection{Fundamento axiológico}

Se refiere a los valores sociales, investigativo, en cuanto a su producción de conocimiento para aportar a validez a las complejas situaciones que presenta una sociedad tan cambiante, ese valor del ser humano en afrontar realidades, Barberousse (2008), propone que:

El ser humano ha logrado interactuar de una manera eficiente, y cada vez más inteligente, con su entorno, y aumentó su capacidad de reflejar, creativamente, la realidad por medio de cálculos y modelos formales (pág. 97).

En la actualidad esta similitud que lleva a una sociedad especialmente las comunidades de escasos recursos económicos, para satisfacer necesidades básicas de una familia para aumentar su capacidad de responder a sus adversidades.

\subsection{Fundamentos epistemológicos}

Lo epistemológico se relaciona con el conocimiento de la realidad social, con lo objetivo y subjetivo, su origen y naturaleza epistémica de la investigación científica, en palabras de Morín (1994): pueda considerar nuestro propio conocimiento como objeto de conocimiento, es decir que este conocimiento a través de distintos puntos de vista como la mirada social de la comunidad y la mirada epistémica debe ser crítica, autocritica y reflexiva propio de la transcomplejidad.

\subsection{Fundamentos Metodológicos}

La fundamentación metodológica que sustenta la transcategorial emergente de la seguridad ciudadana es la transdisciplinariedad, que permite la interconexión de conocimiento multidimensional entre la comunidad y universidad, afianza la vinculación social con la organización, reorganización, planeación en contante dialogicidad. 


\section{Metodología}

En toda investigación científica, es necesario que los hechos estudiados, su interrelación y los resultados de la indagación, sean confiables y objetivos, por lo que se requiere realizar la delimitación de los procesos metodológicos que intentaran responder a las interrogantes surgidas, con el objeto de estudio. En ese transitar de diálogos y transdisciplinariedad metodológica, el investigador expone en este artículo la Metodología Transcompleja, como base epistémica principal en la investigación. Al respecto, González, et al. (2018), concatena que:

La investigación desde el enfoque transcomplejo, aboga por la inclusión de todos los recursos disponibles que le permitan transitar por senderos inexplorados hasta ahora por los paradigmas vigentes; esto significa que la transcomplejidad asume la investigación más allá de los paradigmas es por ello que admite la pluralidad de significados en la ruta que la conduce al conocimiento donde procura la fusión interparadigmática necesaria para obtener su fin último crear nuevas oportunidades y distintas alternativas (pág. 71).

Sin embargo, dentro de nuevas oportunidades y alternativas en la metodología transcompleja permite desarrollar una interrelación epistémica multidimensional, es decir que en la investigación desde el enfoque cuantitativo, cualitativo y dialectico se complementan para buscar la verdad de un problema de estudio. La metodología transcompleja es aplicada en esta investigación porque es abierta a otros métodos de investigación como es el caso de la Investigación Acción Participativa (IAP) y Hermenéutica Crítica.

El desarrollo de esta investigación se orienta bajo el enfoque transcomplejo, de acuerdo a Bracho (2012): como una "verdadera cultura investigativa necesaria para el fortalecimiento de la investigación, en cuanto a la complejidad y niveles de exigencia [...]" (pág. 65). En tal sentido esta forma de pensamiento crítico y creativo debe ser integral entre lo práctico, lo vivencial, y emocional. Señalando a Zaa (2015), citado por Villegas y Alfonzo 
(2017): manifiesta la necesidad de entender la Transcomplejidad:

Como un ejercicio de pensamiento mediante el cual se crean espacios de reflexión donde se entrelazan las percepciones objetivas y subjetivas de la realidad, sus dimensiones lineales y multidimensionales, las concepciones universales y multiversales, lo aparencial y lo substancial, la naturaleza y la artificialeza, lo esencial y lo existencial, lo inductivo concreto y lo deductivo abstracto, lo material y lo energético; en fin lo racional y lo vivencial; como un intento de conformación de novedosos y variados paisajes cognitivos, que contribuyan a la construcción de inéditos e inexplorados caminos del conocimiento humano... (pág. 126).

Un pensamiento inacabado, capaz de tener una visión universal, transdimensional, es lograr crear caminos o senderos de conocimientos para entender y aportar episteme inéditos a la seguridad ciudadana. En lo relativo a técnicas de recolección de datos, como dice Pérez (2015a): "la mayoría de los especialistas coinciden en clasificarlas en dos grandes grupos. Uno referido a la observación, la observación participante y no participante, y el otro relacionado con la entrevista a profundidad, los informantes claves y la entrevista semi-estructurada" (pág. 142); ante esta referencia cualitativa, esta investigación utilizará las siguientes técnicas:

Observación Participante: consiste en la observación del contexto desde la participación directa del investigador y la comunidad a objeto de estudio. Para Valles (1999), citado por Pérez (2015b): "suele entenderse por "observación" los procedimientos en los que el investigador presencia en directo el fenómeno que estudia" (págs. 142-143); es decir que el investigador tiene contacto directo con el problema estudiado. De este modo la presente investigación tiene su epicentro en la Universidad Nacional Experimental de la Seguridad (UNES), estado Aragua.

Encuesta: se desarrollará de forma abierta y cerrada, como medio de interacción individual y grupal. De acuerdo con Arias (2012): "es una estrategia 
(oral o escrita) cuyo propósito es obtener información: a). Acerca de un grupo o muestra de individuos [...] b). En relación con la opinión de éstos sobre un tema específico [...]" (pág. 32). En este sentido, Palella y Martins (2012a): dicen que representa "una técnica destinada a obtener datos de varias personas cuyas opiniones interesan al investigador. [...] Se utiliza un listado de preguntas escritas que se entregan a los sujetos quienes, en forma anónima, las responden por escrito" (pág. 123). El Cuestionario, por su parte, es definido por Palella y Martins (2012b): como "[...] el investigador se debe plantear una serie de cuestiones: tipo de cuestionario que va a utilizar; valoración del tipo de preguntas a incluir [...]" (pág. 133); de igual forma "está obligado a responder a un orden lógico. Las preguntas serán agrupadas por temas afines, en concordancia con una secuencia en orden de dificultad creciente" (pág. 134).

Informantes clave: son un conjunto de personas con ciertas características fundamentales para la investigación, Pérez (2015c), revela que: "son conocidos como actores sociales que proporcionan las pesquisas requeridas para el acercamiento al objeto de estudio" (pág. 147).

Categorización: Nos permite en la investigación explorar la información obtenida de los informantes clave de la comunidad el Progreso. Acorde con esto, Strauss y Corbin (2002), citados por Pérez (2015d): la definen como "el proceso analítico por medio el cual se fragmentan, conceptualizan e integran los datos para formar la teoría" (pág. 152).

Triangulación: para la investigación esta técnica es utilizada para aclarar significados y contrastar diferentes métodos y teorías para crear nuevas teorías. Mencionando a Pérez (2015e):

Las dos grandes fortalezas de este método se encuentran, por una parte, en que logra disminuir los márgenes de error, y por otra en que los resultados obtenidos presentan más confiabilidad cuando se aplican varios métodos o fuentes de información en el análisis de la información (pág. 154). 


\section{Artículo Original / Original Article}

\section{Resultados}

Nuestros aportes con los nuevos conocimientos transcomplejos sobre la vinculación social y la seguridad ciudadana es considerada en las siguientes conceptualizaciones teóricas:

Gráfico 1. Matriz Epistémico de la Investigación Acción Participativa Transcompleja (IAPT).

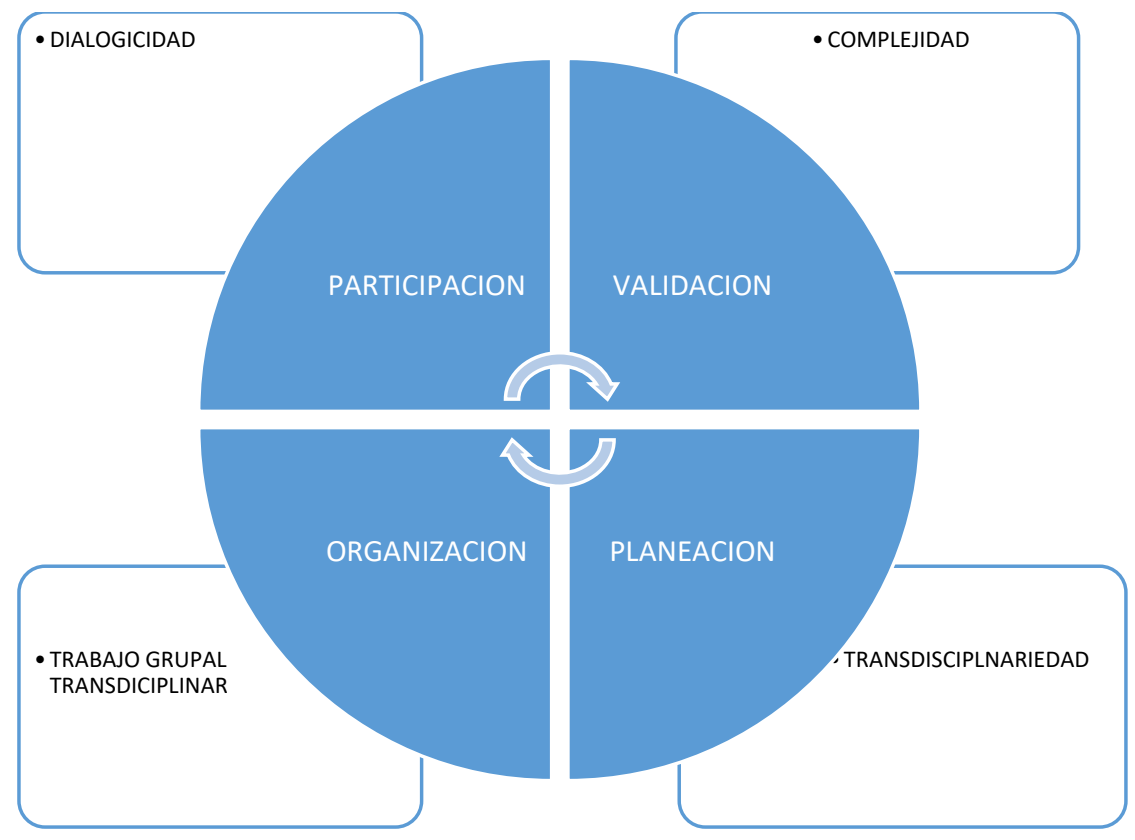

Fuente: El Autor (2020).

Como se puede visualizar en el gráfico 1. La Dialogicidad: entendiendo esta dialogicidad, como el principal dialogo de saber popular, que existe en la comunidad y universidad. Transdisciplinariedad: permite en intercambio de disciplinas que articulan en el mismo objeto de estudio. Caso (Seguridad Ciudadana), Complejidad: Nos encamina a ver lo contraste de la realidad social, (Orden-Desorden). Organización: Permite delinear el trabajo comunitario, perfilando la Planeación activa de los objetivos estratégicos a desarrollar, Participación accionar comunitario e institucional involucrados con el fin de avanzar en el Trabajo Grupal transdisciplinar (incorporando: 
psicólogos, abogados, educadores, cultores, trabajadores sociales, entre otros) y por último la Validación cumplirá la revisión y reflexión de los procesos.

\subsection{Seguridad Ciudadana}

Políticas públicas construida sobre la base de la evolución del pensamiento, actuar en conjunto con los elementos constituyentes en atención del ser humano, para fortalecer la necesidad de convivir en armonía la ciudadanía y potenciar los derechos individuales y colectivos.

\subsection{Vinculación Social}

Eje transversal, inter relacional con principios de dialogicidad, emancipación, en articulación de conocimientos con las organizaciones sociales capaz de reconstruir elementos inherentes en materia de seguridad ciudadana, para transformar la realidad y satisfacer necesidades.

\section{Conclusiones}

La complejidad de la vida, la sociedad, el ser humano, el sistema que nos arropa y desarropa, es menester presentar a consideraciones aportes científicos que surgieron de esta investigación considerando fundamentalmente los principios de la complejidad y transdisciplinariedad de las cuales podemos mencionar:

La transcomplejidad como paradigma de educación para generar nuevos conocimientos. Concluye que la formación profesional debe ser estudiada por distintos enfoques desde la complejidad y la transdisciplinariedad, para garantizar una optimización de Seguridad Ciudadana.

Con respeto a la caracterización del estado actual de la vinculación social, refleja una perspectiva simplista y reduccionista. Los elementos de la transdisciplinariedad en la vinculación social ameritan ser expuestos y 
profundizar las ciencias sociales, jurídicas y humanas, como eje transversal de la formación de la vinculación social.

Desde el aspecto hermenéutico de la construcción de conocimientos teórico-transdisciplinar de la vinculación social, amerita la necesidad de interarticular con principios de diálogos y reflexibilidad con las organizaciones sociales capaces de reconstruir elementos inherentes en materia de seguridad ciudadana.

\section{Referencias}

Alfonzo, N., Castillo, C., León, R., Quintana, J., Salcedo, Y., Schavino, N., ... Zaa, J. (2018). Teorizando la Transcomplejidad. Primera, edición, ISBN: 978-620-2-14407-0. Letonia, Unión Europea: Editorial Académica Española.

Arias, F. (2012). El Proyecto de Investigación: Introducción a la metodología científica. 6 $6^{a}$ Edición, ISBN: 980-07-8529-9. Caracas, Venezuela: Editorial Episteme, C.A.

Barberousse, P. (2008). Fundamentos teóricos del pensamiento complejo de Edgar Morin. Revista Electrónica Educare, XII(2), 95-113, e-ISSN: 1409-4258. Recuperado de:

https://www.redalyc.org/articulo.oa?id=194114586009

Bracho, K. (2012). Cultura investigativa y producción científica en universidades privadas del municipio Maracaibo del estado de Zulia. REDHECS: Revista electrónica de Humanidades, Educación y Comunicación Social, 7(12), 50-69, e-ISSN: 1856-9331. Recuperado de: https://dialnet.unirioja.es/servlet/articulo?codigo=4172363

Constitución de la República Bolivariana de Venezuela (1999). Capítulo III. De Ios Derechos Civiles. Gaceta Oficial Extraordinaria N ${ }^{\circ} 36.860$ de fecha 30 de diciembre. Caracas, Venezuela: Asamblea Nacional Constituyente. 
González, E., Morillo, D., Mejías, M., González, C., Arévalo, G., Guillén, A., ...

Ramírez, A. (2018). Complementariedad Paradigmática o Metodológica. Primera Edición, ISSN: 1690-3064. San Joaquín de Turmero, Venezuela: Fondo Editorial de la Universidad Bicentenaria de Aragua (FE-UBA).

Morín, E. (1994). Introducción al Pensamiento Complejo. (Trad. del fr. Por Marcelo Pakman). Barcelona, España: Editorial Gedisa.

Palella, S., \& Martins, F. (2012a,b). Metodología de la Investigación Cuantitativa. 1ra reimpresión, ISBN: 980-273-445-4. Caracas, Venezuela: Fondo Editorial de la Universidad Pedagógica Experimental Libertador - FEDUPEL.

Pérez, A. (2015a,b,c,d,e). Guía Metodológica para Anteproyectos de Investigación. $4^{a}$ Edición, ISBN: 980-273-422-5. Caracas, Venezuela: Fondo Editorial de la Universidad Pedagógica Experimental Libertador - FEDUPEL.

Rumazo, A. (2006). Simón Bolívar (Bibliografía). Ediciones de la Presidencia de la República. ISBN: 980-03-0362-6. Caracas, Venezuela: Ministerio del Despacho de la Presidencia.

UNES (2010a,b,c). Documento Fundacional. Venezuela: Universidad Nacional Experimental de la Seguridad (UNES).

Villegas, C., \& Alfonzo, N. (2017). Cultura investigativa en los estudios de postgrado desde el enfoque integrador transcomplejo. Chakiñan, (3), 122-137, e-ISSN: 2550-6722. Recuperado de:

\section{https://doi.org/10.37135/chk.002.03.09}

Venegas, B. (2019). La Motivación (según la Teoría de Maslow) y el hábito lector en los estudiantes del segundo grado de secundaria en la I.E. "Sagrado Corazón de Jesús" del Cusco. Tesis. Lambayeque, Perú: Universidad Nacional Pedro Ruiz Gallo. Recuperado de: http://repositorio.unprg.edu.pe/handle/UNPRG/8162 


\section{Luis Enrique Rodríguez Velazco}

e-mail: abg.luisrodriguez@gmail.com

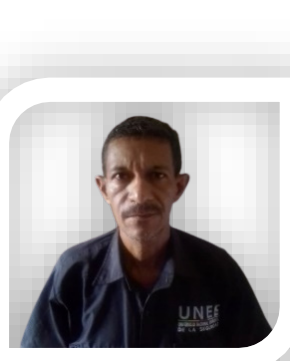

Nacido en Maracay, Venezuela, el 2 de noviembre del año 1970. Profesional administrativo de la Universidad Nacional Experimental de la Seguridad (UNES); abogado de la República Bolivariana de Venezuela; Doctorante en Seguridad Ciudadana en la Universidad Nacional Experimental de la Seguridad (UNES); Diplomado en Desarrollo Comunitario; Seminario en Seguridad Ciudadana y la Nación; Trabajador Social; y actualmente motivado a la investigación de carácter transcomplejo.

El contenido de este manuscrito se difunde bajo una Licencia de Creative Commons ReconocimientoNoComercial-Compartirlgual 4.0 Internacional 